\title{
Designing in the IoT Era: role and perspectives in design practices
}

\author{
Arquilla, Venanzio ${ }^{a}$ \& Vitali, Ilaria ${ }^{b}$ \\ ${ }^{a}$ Design Department - Politecnico di Milano, Italy. venanzio.arquilla@polimi.it. \\ b Design Department - Politecnico di Milano, Italy. ilaria.vitali@polimi.it.
}

\begin{abstract}
In the IoT Era technology is ubiquitous (Kuniavski, 2010) and redundant; in 2009 for the first time in history the number of devices connected to the internet surpassed the number of humans on earth initiating Internet's first true evolution (Evans, 2011).
\end{abstract}

New waves of tech gadgets populate the global markets and the big players are competing with startups and DIY people to commercialize the most innovative and efficient gear.

Thanks to digital fabrication and open source culture smart connected objects can be easily prototyped and sometimes crowdfunded (Jenkins, Bogost, 2014): yielding a Babel of blackboxed, plastic, gadget-like products and services, a first experiment of what is possible and feasible, that in some ways could be defined as an avant-garde trend.

But what about users and their lives? What about the utility and meaning of these objects in real life, with their material qualities and agency of interactions?

This paper, with a bottom-up approach, reflects on a case study in which we started to analyze connected products, reflecting on how technology can "enchant" (Rose, 2014) and augment a smart object while giving value to its tangible part.

The results demonstrates that design can define a balance between tangible and intangible functions of IoT devices, making them desirable, providing new meanings and functions through its physicality, and reinterpreting traditional artifacts.

Some consumers/prosumers are progressively accepting these new connected devices, monitoring health, behaviors and the environment around us, creating big data and modern scenarios for products and services (Acquity Group, 2014).

Physical products pervasively gain a new dimension made of intangible digital avatars (Semmelhack, 2013) able to be freely updated and offer different experiences.

However, technology is often seen as the core of these smart products, resulting in first naive solutions that merely apply electronic components and wireless capabilities to existing objects and in which designers, if present, only manage the aesthetic part. This is an opportunity to apply Design methods and tools to create advanced desirable scenarios for technological objects, bringing users and their interactions back to the core the product development.

Design practices are applied to mediate between users' behaviors and technology, generating devices that leave the gadget-dimension of useless accessories and create more involvement. 
Designing in the IoT era: role and perspectives in design practices.

This means changing the actual design perspective adding new skills and attitudes useful to design research, design education and for professional practices.

Keywords: Internet of Things, design, research, human centered design, tangible interaction, metadesign

\section{The big world of IoT: from infrastructures to everyday lives}

In 1991 Mark Weiser, coiner of the term Ubiquitous Computing, and his team at Xerox Palo Alto Research Center, were already wondering what it would be like to live in a world where computers are seamlessly embedded in all kinds of everyday objects (Weiser, 1991). With the Internet of Things (IoT), not only this is common, but connectivity is also ubiquitous, and devices become digitally identifiable, sensory and context-aware, connected and able to interact with each other in a network of networks. Cisco IBSG affirms that IoT was actually born between 2008 and 2009, the point in time when more "things and objects" were connected to the Internet than people (Evans, 2011). This can be considered as the first real evolution of the Internet, a shift from an Internet of People to one of Things. Our techno-culture crossed the "line of no return" (Sterling, 2005) and is now entering an Internet of Everything, in which People, Things, Data and Processes are connected, combined together to create new capabilities, richer experiences and business opportunities (Evans, 2012): the value of the created network is amplified with each new connection.

With IoT instead of having a small number of powerful devices is possible to have a large number of less powerful devices monitoring different aspects of everyday life (McEwen, Cassimally, 2014), and future forecasts estimates 25 to 50 billion of connected devices in 2025 (Evans, 2011). The potential economic impact is massive, $\$ 4$ to $\$ 11$ trillions a year in 2025 (McKinsey Global Institute, 2015) and Big Players are finally entering the Business to Consumer market with smart home solutions, wearable and nearables, competing with start-ups to commercialize new products.

Everything is Big in the IoT world: (i) the forecasted numbers of connected devices and future economic value; (ii) the size of investments impacting all vertical markets, ranging from cities that want to get smarter to enterprises that seek automatization and efficiency; (iii) data become big, fast and unstructured; (iv) the number of players developing new solutions and products, including Big Players, start-ups and DIY people; (v) and lastly the hype around IoT, which is seen as one of the most important future drivers for innovation.

Debates around IoT mostly gravitate towards two topics: technological challenges and market projections. On one hand, it is relevant to identify what will enable an IoT diffusion and how to deploy it, connecting devices together and to the cloud, achieving interoperability and energy efficiency, gathering and processing big data. On the other hand, economic forecasts help to see where enterprises and cities are investing, what the most promising markets are, and to check if people are ready to buy the newest tech solutions (Osservatorio Internet of Things, 2015). 
Technologists mostly argue about privacy and security implications of cyber-physical devices, generally highlighting that the potential benefits that technology and connectivity may bring to the final users outmatch the concerns. It's evident the value that adding connectivity, sensing capabilities, "intelligence" and automation can deliver when applied at infrastructure level, in smart cities, smart building and enterprises, for smart metering, asset management, logistics, agriculture and many other applications. A better use of existing assets, saving money, resources and energy, increasing efficiency, enabling remote control and monitoring, running pattern recognition algorithms, visualizing predictive analytics in real time are clear benefits of Machine to Machine interaction.

It's less clear how exactly IoT will add worth on the smaller, domestic scale, through smart homes and cars, fitness trackers, wearables and nearables. The first waves of domestic networked products are just an example of what smart things may be; the results are often experiments in which internet capabilities are added to traditional objects, or smartphone-controlled plastic gizmos with many functions. As new connected devices appear in the global distribution and start to become mainstream, new challenges arise for product designers, who need new competences and knowledge to deal with the complexity of this kind of cyber-physical devices.

For modern designers, the IoT should be considered like a mix of enabling technologies to be applied when they are able to add a visible value, acting as an enabler, mediator or facilitator of new services (Cambridge Service Alliance, 2015). Including electronic components and network capabilities in a traditional product is not enough to make it smart, the whole system needs to be designed to ensure a clear and strong value proposition, address real user's needs and guarantee a natural and seamless interaction, so that end users should not need to focus on its connectivity or onboard computing: it should just make sense. (Rowland, et al, 2015)

\subsection{IoT as an entropic system}

Clive Humby first said in 2006 that "Data is the new Oil" (ANA Senior marketer's summit, Kellogg School) of digital economy as objects are connected, can gather real-time signals and accumulate Big Data. Big Data. Big Data is called such because of the large volume of structured and unstructured data that flows and inundates a business on a day-to-day basis. Like oil, it needs to be processed to reveal its true value, transforming raw fragments into useful information. Companies that will gain the most out of IoT are using mathematics (Arthur, 2016) e.g. machine learning algorithms to recognize usage patterns, identify critical situations, predict future development, and enable systems to adapt in real time. Information leads to fast, informed decisions, which can be automated or support users with objective insights. However, currently most IoT data is not used; For example only $1 \%$ of data from an oil rig with 30,000 sensors is examined. Data is mostly used for anomaly detection and control and not yet applied for optimization and prediction (McKinsey Global Institute, 2015).

For now, data are mostly created by things, for things, not exploited at their full potential in an entropic system in which are accumulated. Most of the value is lost without any findings. The IoT Industry itself is a self-sustained system that doesn't communicate its benefits well to end users. In an ideal process, data should be transformed into information, information into actions and knowledge, knowledge into wisdom and experience. To achieve its full potential, IoT should exit the Machine2Machine-only loop and offer ways to consume data usefully and flexibly, e.g. by delivering the right information at the right time to the right user, shifting from an "If This, Then That" approach to an "If This, Then What?" one (Biron, Follett, 2016).

Smart devices now offer an unthinkable level of Omniscience (Rose, 2014) about the world around us, our bodies and behaviors, and the full potential still needs to be grasped. When we move from an entropic 
system to an open -sharing- one, it is possible to envision services in which existing assets and resources can generate new businesses and opportunities. Airbnb and car sharing services are just an example of a virtuous combination of things, people, data and processes.

Instead of Oil, data should become the new Steel (Semmelhack, 2013), a resource used to build upon, to make products and service better and updated, altering the traditional lifecycles to more of an ongoing flow, a kind of living relationship (Biron, et al, 2016).

\section{Designing Smart Connected products}

When the word "design" is used in an Internet of Things discussion, it would probably be in regards of technical details and solutions. For instance, how to design a whole technological infrastructure, how to connect a great number of sensors in an efficient network or code for interoperability and so forth.

In this paper the term design refers to the Industrial Design discipline, as a "trans-disciplinary profession that harnesses creativity to resolve problems and co-create solutions with the intent of making a product, system, service, experience or a business, better". As the International Council of Societies of Industrial Design defines it, "it links innovation, technology, research, business and customers to provide new value and competitive advantage across economic, social and environmental spheres" (ICSID, 2015).

Industrial Design applies methods from different disciplines with a strategic approach, to place humans at the center of the design process and to deliver solutions that are meaningful and desirable for users and for the market. Therefore, how does IoT impact the Industrial Design field? How can designers deal with modern smart products?

There are two fundamental elements that impact the traditional product design approach: first, objects become more technological and connected, and second, technologies become more accessible. The most relevant difference between traditional products and connected devices is that the latter are cyber-physical systems with a double identity: there is the tangible product and its digital counterpart, an avatar of the object liberated of its material form (Semmelhack, 2013). The digital part is often the most significant aspect, used as the main interaction interface to input and visualize data and access all services; tangible interaction is often set aside except for notification purposes. In this scenario the design of products' intangible avatars acquires great importance, to shape the User Experience and plan customer's journey through different media, as users must be free to access the product's functionalities through different touch points in the easiest way. At the same time the physical qualities of materials and analogic interactions must not be left behind in the development of a smart solution.

Another important change is that users now expect connected objects to follow the rules of mobile applications: from products to app-products (Vitali, 2015), platforms for services. Apps are not static, they are usually upgraded with new functions, so products should improve in time too. In the app stores developers are free to market their own solutions. Likewise products should be open platform so that any developer or start-up could extract data and information to build something new. With these changes the nature of the product-consumer relationship will shift deeply: products will evolve even after their shipping through data analysis and continuous user feedback (Biron, Follett, 2016).

Technological components are frequently economical and easier to use. Thanks to prototyping boards, affordable microcontrollers and microprocessors, a huge number of online resources, shared knowledge and communities, makers and designers are able to prototype and develop their own solutions freely. Even manufacturing technologies are conveniently available and allow an almost effortless shift from bits to atoms. 
Given this panorama, what is the role of product designers?

We believe that to grasp this complexity designers need to apply a Metadesign approach to understand technologies, markets and users in order to define products and services that may have a value. Giaccardi and Fisher reflecting on Creativity and Evolution stated that Metadesign is a unique design approach concerned with opening up solution spaces rather than complete solutions (hence the prefix meta-), and aimed at creating social and technical infrastructures in which new forms of collaborative design can take place (Giaccardi, 2008). In our vision this is achieved by evaluation of: (i) Technologies: know what can be developed and humanize technology to build seamless and natural experiences. (ii) Markets: to make the product attractive from an economic and productive point of view, with the right price, positioning and a sustainable, defined production model. (iii) Users: to identify new needs and delineate new meanings. This approach could be compared at the human-centered design process (IDEO, 2015) and is a way to ensure that the final solutions remain connected to the people whom are designed for and for this desirable at the human level, viable at the business level and feasible at the technological one. Metadesign is a design research activity fundamental to identify new subtle unspoken dynamics and achieve meaning-driven innovation (Norman, Verganti, 2012). The output of a Metadesign phase gives designers a broad context in which to define a project brief that will be used to design and test different solutions.

After that will be added: (iv) Product System Service elements, including service and communication aspects, to gain a clear overview of the system complexity, with its stakeholders, touchpoints and relationships. (v) It is also considerably important to design interfaces and the whole phygital interaction (physical and digital) for technological products. (vi) An understanding of manufacturing dynamics and production methods (e.g. DIY, self-production, digital fabrication, mass production).

All above elements frame a wider and complex scope for design. This dimension is also treated on the Advaced Design definition where is stated that Advanced Design is not just highly developed design, but also design which anticipates, which sees before others (Celi, 2014). It is not expected of a single designer to master all those aspects, in fact interdisciplinary collaboration is fundamental in Design and specially in Design for the IoT. Reflecting on the current dynamics of education and professional practices we applied the Metadesign approach in a case study in which, by mapping IoT products and with exhaustive case studies research, we identified a "new" need, a meaning change, that led to the development of a connected product.

\section{Mapping The IoT: research phase and design of InTune - a connected product}

The "Mapping the IoT" project was born as a Master's thesis research at Politecnico di Milano. Our approach was to read the existing Business to Consumer market from a designer point of view, analysing products and trying to find correlations to extract elements that can be later used to design new devices. Since existing classifications are usually generic and mostly focus on market and technological segmentations we felt the need to apply a bottom-up approach inspecting existing consumer solutions to gather data from the market, without imposing any solution. Through the analysis of 107 case studies of smart connected products an Analysis Tool was developed. 

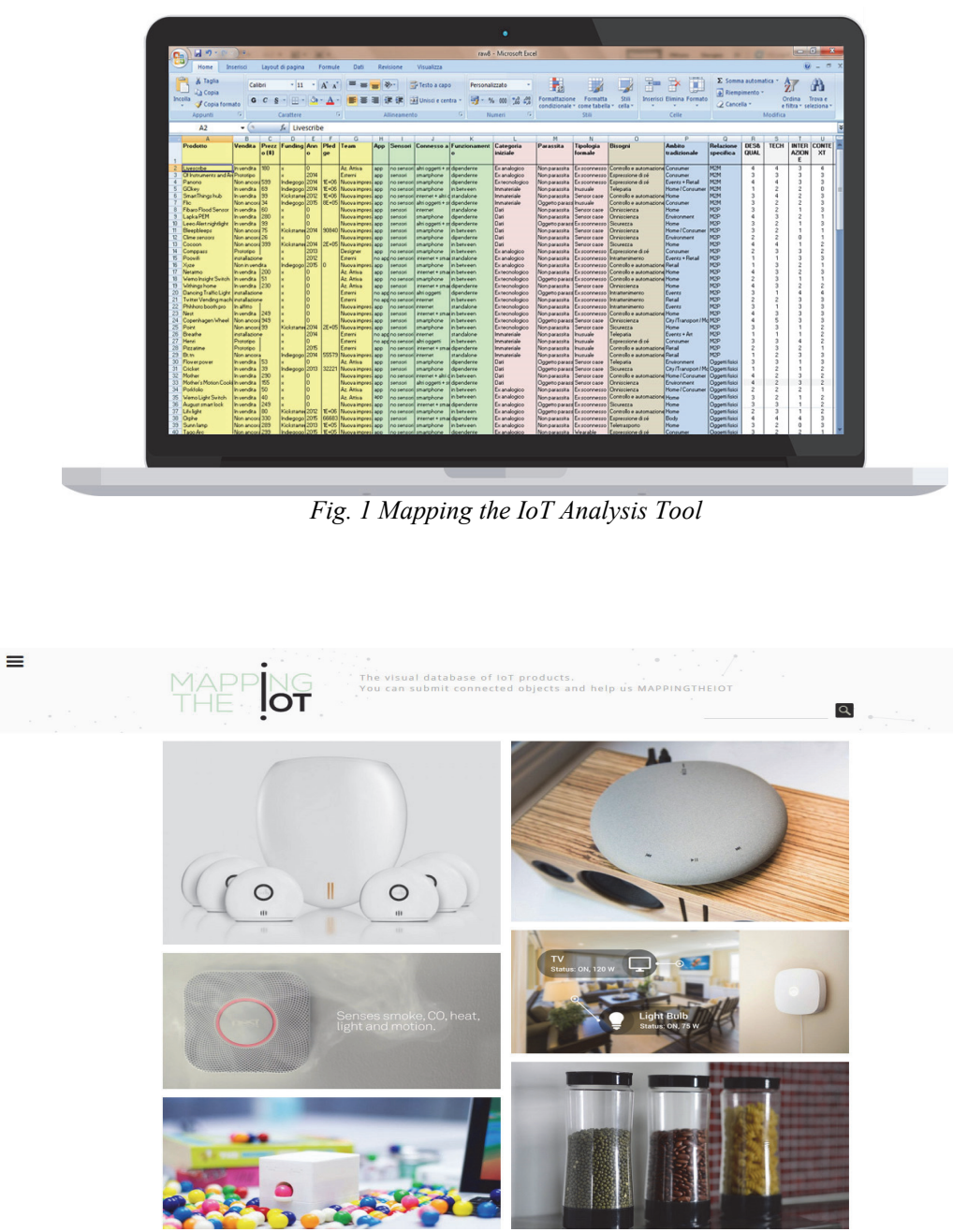

The tool then evolved into an online platform (mappingtheiot.tumblr.com), that can be used as an open research tool, since it simplifies the mapping process through the use of selectable tags and anybody can submit new cases to be reviewed.

Fig. 2 Mapping the IoT, home

summarized as follows: (i) Technological point of view: features and specifications (e.g. sensors, apps, what the product is connected to, relationship and dependence between the product and its apps/the need of connectivity), degree of innovation, area of application. (ii) Financial point of view: e.g. price and perceived value, exploited funding method. (iii) User point of view: what fundamental needs does the product try to fulfil, e.g. omniscience, safety (Rose, 2014), intended user and context (e.g. degree of definition, value of the solution), frequency and quality of interaction. (iv) Design-thinking point of view: conceptual qualities (e.g. is it a new artefact? What is its aim? Can be traced to an archetypical shape/typology of artefacts?)

Of an initial sample of 400 cases, 107 were selected and analysed using the tool. An analysis card was made to map each case study, and data were recorded. 


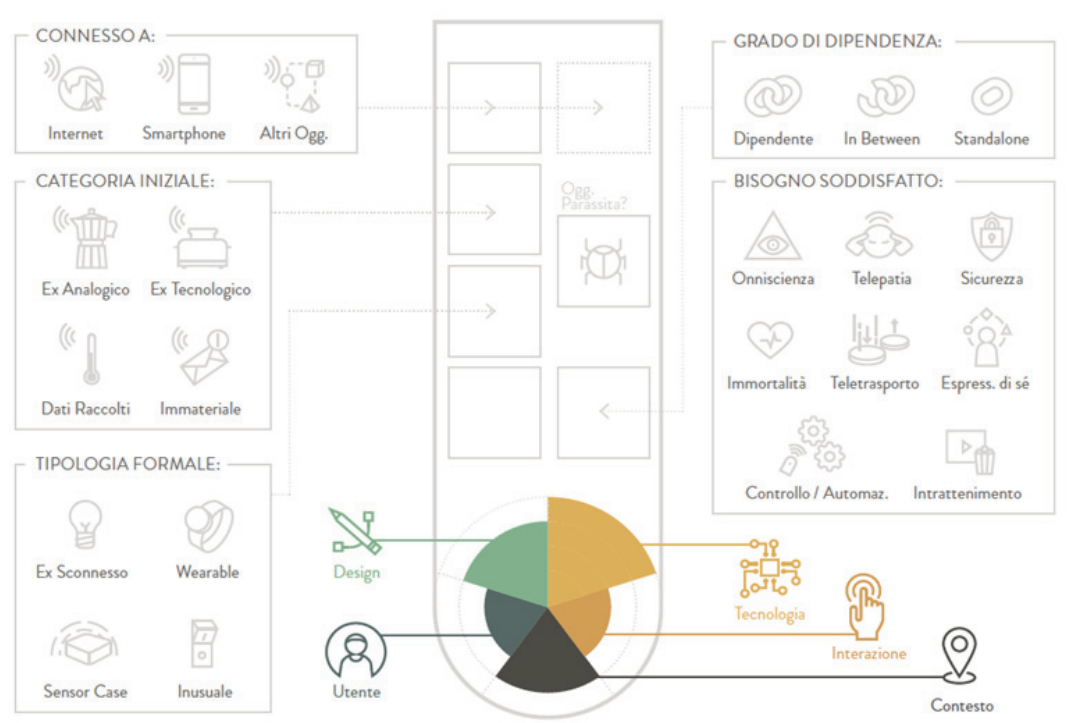

Fig. 3 Legend used to map each case study. Icons and graphs highlight the main characteristics

The gathered data were processed using Raw (raw.densitydesign.org) a versatile online tool that enables data visualization and clustering. The results were summarized in a final report and a set of infographics.

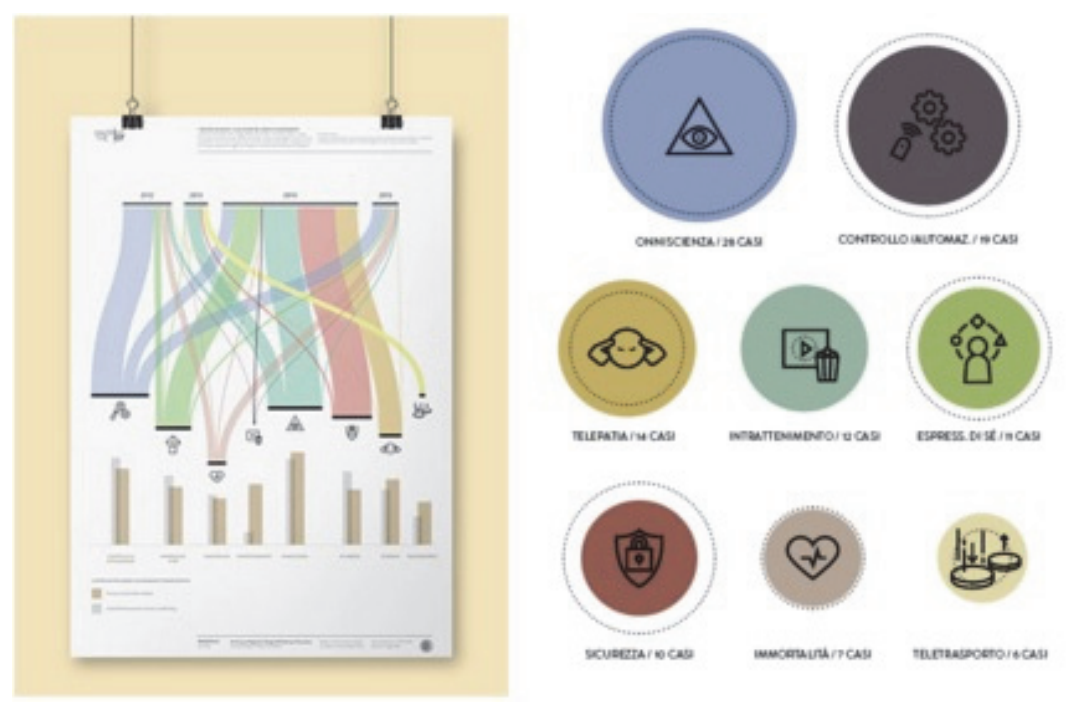

Fig. 4 Mapping the IoT Infographics

From that it was possible to identify features and common characteristics (e.g. the need of omniscience, telepathy, control and automation those products fulfil, the idea to materialize intangible content) that were used as a starting point for the brief definition of InTune, the developed product. 
InTune reflects around the topic of time in our digital modern society. Modern time is managed through shared digital calendars, smart alarms, notifications, personalized objectives and deadlines. In this technological era clocks are not enough to express this complexity and seconds are not the most relevant means of measure. Depending on what users consider important progress may be used as measurement unit; how many calories were burnt today? How many followers are left to reach 1000? How many to-dos remain in the Trello list at the end of the day? How much until your product gets funded on Kickstarter? How long until summer? Personal time is multi-channelled and punctuated by activities, events, tasks, future plans and self-set objectives that may be physical or digital, offline or online. Following the MappingTheIot analysis framework we understood the fundamental needs the product would have had to fulfil: the need of Omniscience (know everything), Telepathy (see only what is important) and Entertainment (keep users motivated, challenging themselves).

The concept of modern time was linked with progress bars and seek bars, two common elements in the digital UI panorama, with visual properties and intuitive use. Thus the idea was to make the bar tangible, connected and reprogrammable, able to interact with online services and existing applications, showing intangible data with a physical medium.

InTune has three different functions: (i) It is a tangible and connected progress bar, compatible with different services and applications (e.g. digital calendars, fitness tracker apps, IFTTT). It uses its own app to add personal objectives and challenge other people, and it can be used as an alarm clock. In this case the smartphone is used as a data input interface. (ii) It is a timer. No app nor smartphone are needed for this function. (iii) It is a Bluetooth enabled speaker: the motorized progress bar moves in sync with the music and it is possible to physically interact with the played song, going backwards or forwards.

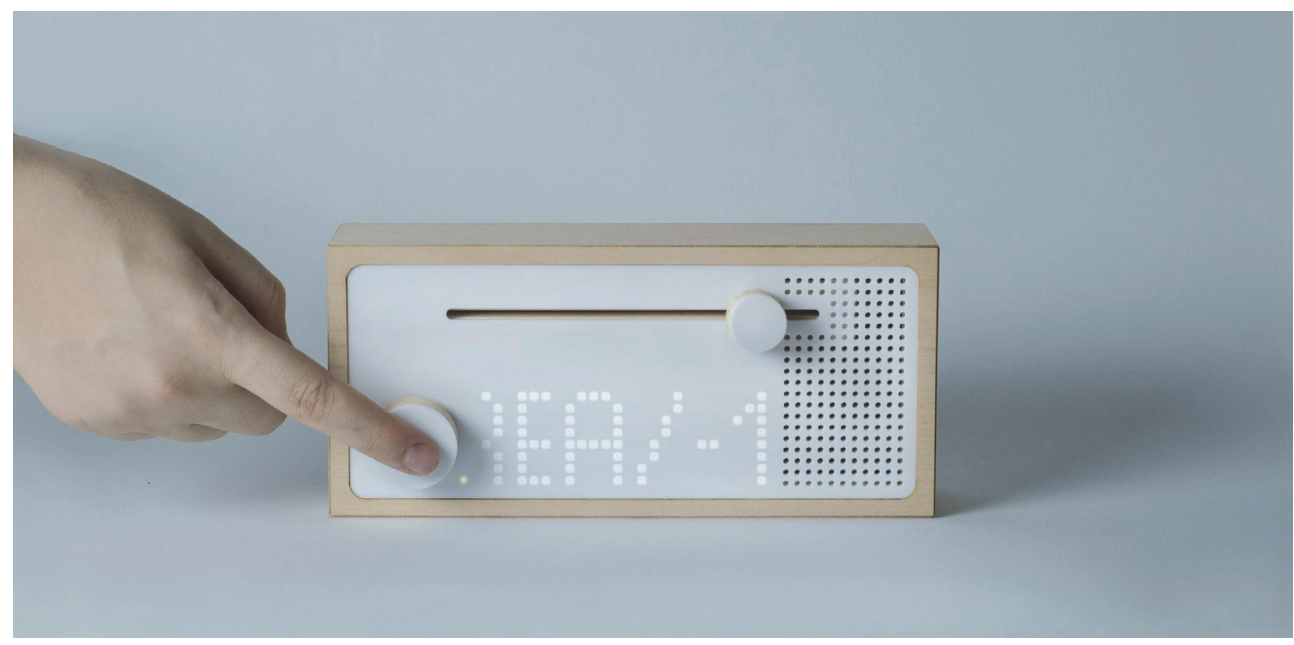

Fig. 5 InTune mockup: display the description of an ongoing progress

All these functions were defined considering the degree of dependence between the product and its app. Connected products often lose functionalities when the smartphone is out of battery: by augmenting a traditional object (speaker) InTune offers more features. The smartphone is used only to create, save, and send new tasks to InTune. To change mode, visualize the progress of all ongoing objectives, the interaction is completely physical and designed to put the smartphone away. 
InTune was one of the winners of "Next Design Innovation", an open call promoted by Regione Lombardia and Polifactory, the makerspace of the Politecnico di Milano (www.polifactory.polimi.it/), and was prototyped and exhibited at Milan Design Week 2016. The product was partially functioning: due to time, components availability, and knowledge limitations only the speaker function was implemented. In order to assemble a working prototype many compromises had to be reached and, although the final result was well manufactured, the object was changed and detuned.

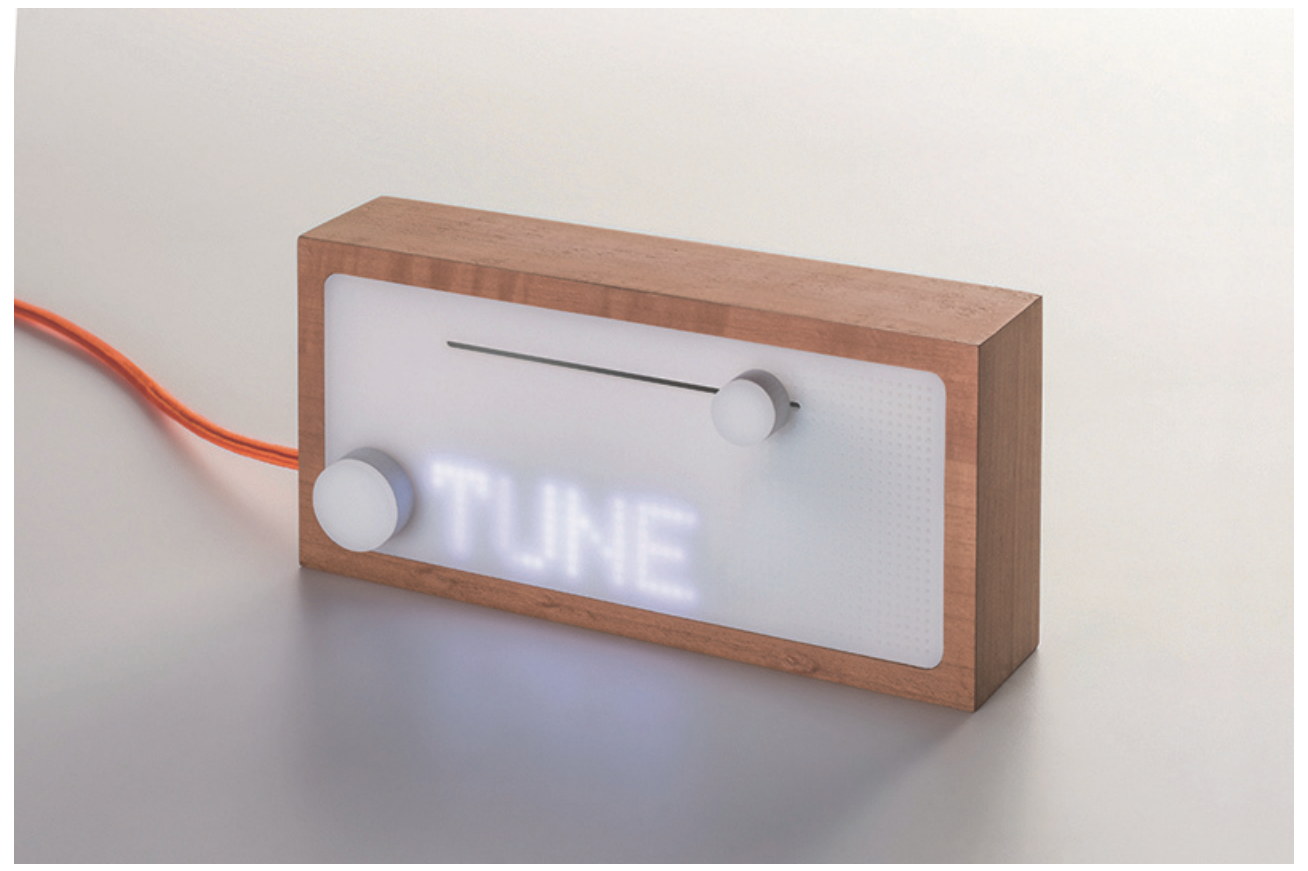

Fig. 6 Next Design Innovation: InTune was prototyped by Polifactory - Politecnico di Milano in collaboration with Regione Lombardia - photo by F. Villa

From this experience we reflected on the topic of prototyping. Designers are given tools to prototype even advanced technological solutions and autonomously materialize their own ideas in new places (Fablab, Makerspace ecc.) where they can also become really Designer=Enterprise (Bianchini, et al, 2014 Arquilla, et al, 2011). We observed that while talking about the Internet of Things it may be an opportunity but also a substantial limitation because designers may be aware of a given conventional technology but unable to put it into practice. Hence the "functioning prototype" self-production threatens to impoverish the concept and qualities of the design scenario, approaching oversimplified solutions but with an acceptable technological complexity. We believe that a line needs to be traced between knowledge and execution, and designers need to focus on the phases in which they can add more value. Technological awareness eventually enables designers to act as "headhunters" and understand what are the needed competences and skills to form a multidisciplinary team to implement any solution, while completing execution

\section{Role and perspectives in design practices}

With the IoT, network technologies are accessible and applicable, the delicate move is to use them consciously, when are really able to add value and innovate. Knowledge is the first step towards consciousness: being aware of what exists and what is feasible empowers designers to conceive new 
scenarios, finding new applications for consolidated technological solutions and generating meaningdriven innovation. The design discipline contributes to give signification to products and services, humanizing technologies, placing people at the centre of the process and exploring their needs to allow a meaningful cultural interpretation and to build compatible captivating services and business models. Hence the IoT could turn into a new important resource in the designers' set of skills.

From our experience we experimented that following a Metadesign approach before starting the design phase may prove an effective way to tackle the design complexity of connected products since both technology, market and user needs are strongly bound together. After our research we constructed a list of guidelines that can be followed while designing for smart connected products, and that frame future perspective in design practices.

(i) Connected products are augmented products: adding network capabilities and sensors, several functions and big data is not enough to make them smart. Meaning needs to be added. From our research it strongly emerged that in the proliferation of technological performance-driven products (Morozov, 2013) there is substantial space to build sense and acceptance, defining innovative dynamics where objects enable services and new interactive interactions.

(ii) Therefore having an app linked to a product is not enough. Applications, network capabilities and sensors are just tools to be wisely exploited: inevitably adding them to any object means to potentially offer a new service. In "Designing for connected products" the authors (Rowland, et al, 2015) state that there may be service-enabled devices and device-enabled services. In the first case the device is seen as the most salient part of the service, central to the overall User Experience (e.g. Nest thermostat); in the second case users just want to benefit from specific functionalities and services (e.g. an alarm service). In both situations there will be tangible objects with both physical and digital aspects. The intangible, digital part may improve flexibly over time, extending the purpose of the tangible product and engaging the final users and it developers in a relationship. It is again crucial to underline that adding connectivity must augment a given object. A notable example of a smart IoT device is Amazon's Kindle, since it augments a traditional artifact (the book) amplifying its strengths and decreasing its weaknesses (one "smart-book" has multiple texts, fast translations, smart notes ...), and connectivity enables a new service: instant book purchase and delivery. Therefore with IoT, objects may not be just bought but have different business models, inspired by digital business models (e.g. freemium and premium model) or service ones (e.g. sharing). It is evident that connected devices are strongly linked with the servitization phenomenon, which is the process of creating value by adding services to products (Cambridge Service Alliance, 2015).

(iii) The value proposition of complex product has to be clear: users need to perceive how connectivity can unlock new services and meanings. Performances may be simpler or limited but designers need to create beneficial and appealing use scenarios that clearly communicate the worth of a proposed solution. It is then interesting to point out that early adopters of IoT solutions are enjoying their wearable devices more than the apps they use in conjunction with (Breitenfeld, 2016) because there is a gap between what objects pledge and what they really deliver. Therefore creating a scenario is not enough, designers must supervise and ensure that there is consistency between the value proposition and the effective execution. This also means trying to design how users can truly make use of the data that many smart devices produce.

(iv) Connected products are phygital, but that does not mean that screens and smartphones should be the only way to interact with them. Depending on the project one aspect may be more relevant than the other, still we advise to design for balance between tangible and intangible features. Since functions often become intangible it is indeed important not to overlook material and sensorial qualities and physical 
interaction with artefacts and devices. The physicality of products has to be managed with quality and regain its centrality.

Going back to Weiser again, he stated that "the most profound technologies are those that disappear. They weave themselves into the fabric of everyday life until they are indistinguishable from it" (Weiser, 1991). Thus the role of design is supporting this seamless combination, using design-related tools and practices, specially the Metadesign one, to envision and materialize novel products and processes giving them quality and meaning.

\section{References}

AA. VV., (2016). Next Design Innovation, Libraccio Editore, Milano

ACQUITY GROUP. (2014). The Internet of Things: The Future of Consumer Adoption. Acquity Group's 2014 Internet of Things Study. $<$ http://quantifiedself.com/docs/acquitygroup-2014.pdf> [See: February26, 2016]

ARDUINO. (2016). Arduino Iot Manifesto. $<$ https://create.arduino.cc/iot/manifesto/> [See: April 27, 2016]

ARQUILLA V., MAFFEI S., BIANCHINI M. (2011), Designer = Enterprise. A new policy for the growth of the next Italian design.. pp.177-184. In Design Management. Toward a new era of innovation. - ISBN:9789881598417

ARTHUR, R. (2016). Data Is the New Oil' and More from SXSW. Business Of Fashion, March 16 $<$ http://www.businessoffashion.com/articles/fashion-tech/data-is-the-new-oil-and-more-from-sxsw $>$ [See: May 5, 2016]

BIANCHINI, M., BOLZAN, P., MAFFEI, S. (2014). (re)Designing Design Labs. Processes and places for a new generation of Designers $=$ Enterprises. Nord Design 2014, Conference Prodeedings, August 27 - 29, 2014 Espoo, Finland

$<$ https://www.academia.edu/8629200/re Designing_Design_Labs. Processes_and places_for_a_new_generation_o f_Designers_Enterprises $>$ [See: May 5, 2016]

BIRON, J., FOLletT, J. ( 2016). Foundational Elements Of An Iot Solution. Sebastopol: O’Reilly Media, Inc. $<$ http://www.thingworx.com/White-Papers/IoT-OReilly-Foundational-Elements-for-IoT-eBook-

1?utm_campaign=IoT\%20ThingWorx\%20Newsletter\%20April\%20Email\%203B\&utm_medium=email\&utm_ssurce $=$ Eloqua \&src $=$ TSbutton $1 \&$ elqTrackId $=4 \mathrm{a} 6 \mathrm{cac} 2 \mathrm{cb} 8754 \mathrm{~d} 0 \mathrm{fa} 85 \mathrm{da} 6 \mathrm{c} 90 \mathrm{~d} 02 \mathrm{e} 661 \& \mathrm{elq}=28 \mathrm{~d} 896 \mathrm{a} 154364 \mathrm{~b} 72 \mathrm{a} 62 \mathrm{e} 5 \mathrm{a} 0043 \mathrm{~b}$ 5d412\&elqaid=16429\&elqat=1\&elqCampaignId=2771 $>$ [See: April 27, 2016]

BREITENFELD, M. (2016). Examining the Wearables Ecosystem Roadblocks in Personal Data Interpretation. Argus Insights. $<\mathrm{http}: / /$ www.argusinsights.com/wearable-apps-2016//> [See April 26, 2016]

CAMBRIDGE SERVICE ALLIANCE. (2015). The Future of Servitization: Technologies That Will Make A Difference. Cambridge: University of Cambridge. $<$ http://cambridgeservicealliance.eng.cam.ac.uk/resources/Downloads/Monthly\%20Papers/150623FutureTechnologie sinServitization.pdf/view> [See April 27, 2016]

CELI, M., (2014) Advanced Design Cultures: Long-Term Perspective and Continuous Innovation. Springer International Publishing.

EVANS, D. (2011). The Internet of Things : How the Next Evolution of the Internet Is Changing Everything. Cisco Internet Business Solutions Group (IBSG). $<$ http://www.cisco.com/c/dam/en_us/about/ac79/docs/innov/IoT_IBSG_0411FINAL.pdf> [See: February 25, 2016]

EVANS, D. (2012). The Internet of Everything: How More Relevant and Valuable Connections Will Change the World. Cisco Internet Business Solutions Group (IBSG). <http://www.cisco.com/web/about/ac79/innov/IoE.html> [See: February 25, 2016]

GIACCARDI, E., FISCHER, G., (2008), Creativity and Evolution: A Metadesign Perspective, Digital Creativity, 19(1), pp. 19-32. <http://13d.cs.colorado.edu/ gerhard/papers/digital-creativity-2008.pdf> [See April 26, 2016]

ICSID, ICSID Definition of Design. <http://www.icsid.org/about/about/articles31.htm> [See: May 1, 2016]

IDEO, (2015), Design Kit, What is Human-Centered Design? <http://www.designkit.org/human-centered-design> [See: May 10, 2016] 
Designing in the IoT era: role and perspectives in design practices.

JENKINS, T. BOGOST, I., (2014). Designing for the Internet of Things : Prototyping Material Interactions. Toronto, ON, Canada: CHI 2014, One of a CHInd. [two authors]

KUNIAVSKY, M. (2010). Smart Things : Ubiquitous Computing User Experience Design. Amsterdam: Morgan Kaufmann. [an author]

MAPPINGTHEIOT, Mapping The Iot: the visual database of IoT products. $<$ http://mappingtheiot.tumblr.com $>$ [See: Apr. 20, 2016]

MCEWEN, A., CASSIMALLY, H. (2014). Designing The Internet Of Things. Chichester: Wiley [two authors]

MCKINSEY GLOBAL INSTITUTE. (2015). The Internet of Things: mapping the value beyond the hype. McKinsey Global Institute (MGI). <http://www.mckinsey.com/business-functions/business-technology/our-insights/the-internetof-things-the-value-of-digitizing-the-physical-world $>$ [See: February 27, 2016]

MOROZOV, E. (2013). To save everything, click here: technology, solutionism, and the urge to fix problems that don't exist. London: Allen Lane. [an author]

NORMAN, D., VERGANTI, (2012). Incremental and radical innovation: design research versus technology and meaning change. Design Issues $<$ http://jnd.org/dn.mss/Norman\%20\%26\%20Verganti.\%20Design\%20Research\%20\%26\%20Innovation$18 \% 20 \mathrm{Mar} \% 202012 . p d f>$ [See: May 10, 2016]

OSSERVATORIO INTERNET OF THINGS. (2015). Internet Of Things: il futuro è già presente! Report 2015. Milano: Politecnico di Milano: School of Management: Osservatorio Internet of Things

O'Reilly. (2015) Designing For The Internet Of Things: A Curated Collection Of Chapters From The O'Reilly Design Library. O’Reilly Media. <http://www.oreilly.com/design/free/designing-for-the-internet-of-things.csp $>$ [See: May 7, 2016]

RAW. Raw, The missing link between spreadsheets and vector graphics. < Raw.densitydesign.org> [See: May 1, 2016]

ROSE, D. (2014). Enchanted Objects: Design, Human Desire, and the Internet of Things. New York: Scribner. [an author]

ROWland, C., GOODMAN, E., CHARlier, M., LIGHT, A., LUI, A. (2015). Designing Connected Products. O'Reilly Media [five authors]

SEMMELHACK, P. (2013). Social Machines: How to Develop Connected Products That Change Customers'Lives. Hoboken, New Jersey: John Wiley \& Sons Inc. [an author]

STERLING, B. (2005). Shaping Things. Cambridge, Mass.: MIT Press. [an author]

VITALI, I. (2015). Mapping the Iot: un percorso di ricerca, analisi e sperimentazione in ambito Internet of Things. Master's thesis. Milan: Politecnico di Milano.

WEISER, M. (1991). The Computer For The 21St Century. Scientific American, Inc. 1991, Reprinted with permission in IEEE Pervasive Computing. 2002, January-March issue, p. 18-25 <www.cs.cmu.edu/ jasonh/courses/ubicomp-sp2007/papers/02-weiser-computer-21st-century.pdf> [See: May 7, 2016] 